International Journal of Pure and Applied Mathematics

Volume 96 No. 2 2014, 197-212

ISSN: 1311-8080 (printed version); ISSN: 1314-3395 (on-line version)

url: http://www.ijpam.eu

doi: http://dx.doi.org/10.12732/ijpam.v96i2.4

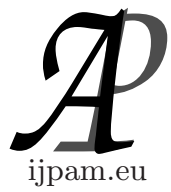

\title{
FIXED POINT RESULTS FOR GENERALIZED RATIONAL TYPE CONTRACTIONS WITH PPF DEPENDENCE
}

\author{
Preeyanud Chuasuk ${ }^{1}$, Anchalee Kaewcharoen ${ }^{2} \S$ \\ ${ }^{1,2}$ Department of Mathematics \\ Faculty of Science \\ Naresuan University \\ Phitsanulok, 65000, THAILAND
}

\begin{abstract}
In this paper, we introduce the certain contractive conditions for mappings which are weaker than rational type contractions and we assure the PPF dependent fixed point theorems in the Razumikhin class for such mappings in Banach spaces. Furthermore, the PPF dependent fixed point theorems in the Razumikhin class for such mappings in partially ordered Banach spaces are proven.
\end{abstract}

AMS Subject Classification: 54H25, 55M20

Key Words: PPF dependent fixed points, Razumikhin classes, algebraic closedness with respect to difference

\section{Introduction and Preliminaries}

The fixed point theory plays an important role and it has been continually studied for decade (see [1], [4], [8], [9], [10], [11], [13], [14], [17] and references contained therein). Bernfeld et al. [2] proved the existence of a PPF (past, present and future) dependent fixed point in the Razumikhin class for a mapping $T$ whose domain is the Banach space of continuous mappings defined on an interval $[a, b]$ with range in a Banach space $E$ and the range of $T$ is in $E$.

Received: March 4, 2014

(C) 2014 Academic Publications, Ltd.

${ }^{\S}$ Correspondence author url: www.acadpubl.eu 
Afterward, there are many researchers assured the existence of PPF dependent fixed point theorems for mappings satisfying the weaker contractive conditions (see, for examples, [4], [5] , [6], [12], [18]). In 2007, Drici et al. [5] proved the PPF dependent fixed point results in partially ordered metric spaces. Dass and Gupta [3] and Jaggi [7] proved the fixed point theorems for mappings satisfying rational contractive type conditions. Recently, Sintunavarat and Kumam [18], proved the PPF dependent fixed point theorems in the Razumikhin class for rational type contractions in Banach spaces.

In this paper, we introduce the certain contractive conditions for mappings which are weaker than rational type contractions and we assure the PPF dependent fixed point theorems in the Razumikhin class for such mappings in Banach spaces. Furthermore, the PPF dependent fixed point theorems in the Razumikhin class for such mappings in partially ordered Banach spaces are proven.

Suppose that $E$ is a Banach space with the norm $\|\cdot\|_{E}$ and $I$ is a closed interval $[a, b]$ in $\mathbb{R}$. Let $E_{0}=C(I, E)$ be the set of all continuous $E$-valued mappings on $I$ equipped with the supremum norm $\|\cdot\|_{E_{0}}$ defined by

$$
\|\phi\|_{E_{0}}=\sup _{t \in I}\|\phi(t)\|_{E},
$$

for all $\phi \in E_{0}$. For a fixed element $c \in I$, the Razumikhin class of mappings in $E_{0}$ is defined by

$$
\mathcal{R}_{c}=\left\{\phi \in E_{0}:\|\phi\|_{E_{0}}=\|\phi(c)\|_{E}\right\} .
$$

Recall that a point $\phi \in E_{0}$ is said to be a PPF dependent fixed point or a fixed point with PPF dependence of $T: E_{0} \rightarrow E$ if $T \phi=\phi(c)$ for some $c \in I$.

Definition 1. Let $K$ be a subset of $E$. Then

(i) $K$ is said to be topologically closed with respect to the norm topology if for each sequence $\left\{x_{n}\right\}$ in $K$ with $x_{n} \rightarrow x$ as $n \rightarrow \infty$ implies $x \in K$.

(ii) $K$ is said to be algebraically closed with respect to the difference if $x-y \in$ $K$ for all $x, y \in K$.

Recently, Sintunavarat and Kumam [18], introduced the definition of rational type contractions and proved the PPF dependent fixed point theorems in the Razumikhin class for such mappings in Banach spaces.

Definition 2. A mappings $T: E_{0} \rightarrow E$ is called a rational type contraction if there exist real numbers $a, b \in[0,1)$ with $a+b<1$ satisfying

$$
\|T \phi-T \alpha\| \leq a\|\phi-\alpha\|_{E_{0}}+\frac{b\|\phi(c)-T \phi\|_{E}\|\alpha(c)-T \alpha\|_{E}}{1+\|T \phi-T \alpha\|_{E}},
$$


for all $\phi, \alpha \in E_{0}$ and for some $c \in I$.

Theorem 3. ([18]) Suppose that $T: E_{0} \rightarrow E$ is a rational type contraction. Assume that $\mathcal{R}_{c}$ is topologically closed with respect to the norm topology and is algebraically closed with respect to the difference. Then $T$ has a unique PPF dependent fixed point in $\mathcal{R}_{c}$.

In 2007, Drici et al. [5] proved the following the fixed point theorems in partially ordered metric spaces for mappings with PPF dependence.

Theorem 4. ([5]) Let $(E, d, \leq)$ be a partially ordered complete metric space and $T: E_{0} \rightarrow E$ where $E_{0}=C(I, E)$. Assume that

(i) $T$ is a nondecreasing mapping;

(ii) for all $\phi, \alpha \in E_{0}$ with $\phi \leq \alpha, d(T \phi, T \alpha) \leq k d_{0}(\phi, \alpha)$ where $d_{0}(\phi, \alpha)=$ $\max _{s \in I} d(\phi(s), \alpha(s))$ and $k \in[0,1)$;

(iii) there exists a lower solution $\phi_{0}$ such that $\phi_{0}(c) \leq T \phi_{0}$;

(iv) $T$ is a continuous mapping or if $\left\{\phi_{n}\right\}$ is a nondecreasing sequence in $E_{0}$ converging to $\phi \in E_{0}$, then $\phi_{n} \leq \phi$ for all $n \in \mathbb{N}$.

Then $T$ has a $P P F$ dependent fixed point in $E_{0}$.

In this paper, we use the following notations.

$$
\begin{aligned}
\Psi_{1}= & \{\psi: \psi:[0,+\infty) \rightarrow[0,+\infty) \text { is nondecresing with } \\
& \left.\sum_{n=1}^{\infty} \psi^{n}(t)<\infty, \text { for all } t \in(0,+\infty)\right\} \text { and } \\
\Psi_{2}= & \{\psi: \psi:[0,+\infty) \rightarrow[0,+\infty) \text { is continuous nondecreasing with } \\
& \psi(t)<t \text { for all } t \in(0,+\infty)\} .
\end{aligned}
$$

Remark 5. Since every nondecreasing mapping is differentiable almost everywhere (see [15]), we observe that the nondecreasing condition is closed to the continuity and it is restrictive.

Lemma 6. [16] Suppose that $\psi:[0,+\infty) \rightarrow[0,+\infty)$. If $\psi$ is nondecreasing, then for each $t \in(0,+\infty), \lim _{n \rightarrow \infty} \psi^{n}(t)=0$ implies $\psi(t)<t$.

Remark 7. (i) It is easily seen that if $\psi:[0,+\infty) \rightarrow[0,+\infty)$ is nondecreasing and $\psi(t)<t$ for all $t \in(0,+\infty)$, then $\psi(0)=0$.

(ii) We can see that if $\psi:[0,+\infty) \rightarrow[0,+\infty), \psi(t)<t$ for all $t \in(0,+\infty)$ and $\psi(0)=0$, then $\psi$ is continuous at 0 . 
In this paper, we introduce the certain contractive conditions for mappings involving two families $\Psi_{1}$ and $\Psi_{2}$ which are weaker than rational type contractions and we assure the PPF dependent fixed point theorems in the Razumikhin class for such mappings in Banach spaces. Furthermore, the PPF dependent fixed point theorems in the Razumikhin class for such mappings in partially ordered Banach spaces are proven. Our results generalized the results proved by $[18]$.

\section{PPF Dependent Fixed Points for Rational Type Contractions in Banach Spaces}

We now prove the PPF dependent fixed point theorems for mappings satisfying the generalized contractive conditions concerning with $\psi \in \Psi_{1}$.

Theorem 8. Suppose that $\psi \in \Psi_{1}$ and $T: E_{0} \rightarrow E$ satisfies the following condition:

$$
\|T \phi-T \alpha\|_{E} \leq \psi\left(\max \left\{\|\phi-\alpha\|_{E_{0}}, \frac{\|\phi(c)-T \phi\|_{E}\|\alpha(c)-T \alpha\|_{E}}{1+\|T \phi-T \alpha\|_{E}}\right\}\right),
$$

for all $\phi, \alpha \in E_{0}$. Assume that $\mathcal{R}_{c}$ is topologically closed with respect to the norm topology and algebraically closed with respect to the difference. Then $T$ has a unique PPF dependent fixed point in $\mathcal{R}_{c}$.

Proof. Let $\phi_{0} \in \mathcal{R}_{c}$. Since $T \phi_{0} \in E$, we can choose $\phi_{1} \in \mathcal{R}_{c}$ such that

$$
T \phi_{0}=\phi_{1}(c) \text { and }\left\|\phi_{0}-\phi_{1}\right\|_{E_{0}}=\left\|\phi_{0}(c)-\phi_{1}(c)\right\|_{E} .
$$

Similarly, since $T \phi_{1} \in E$, we can choose $\phi_{2} \in \mathcal{R}_{c}$ such that

$$
T \phi_{1}=\phi_{2}(c) \text { and }\left\|\phi_{1}-\phi_{2}\right\|_{E_{0}}=\left\|\phi_{1}(c)-\phi_{2}(c)\right\|_{E} .
$$

By continuing the process as before, we can construct the sequence $\left\{\phi_{n}\right\}$ in $\mathcal{R}_{c}$ such that

$$
T \phi_{n}=\phi_{n+1}(c) \text { and }\left\|\phi_{n}-\phi_{n+1}\right\|_{E_{0}}=\left\|\phi_{n}(c)-\phi_{n+1}(c)\right\|_{E},
$$

for all $n \in \mathbb{N} \cup\{0\}$. For each $n \in \mathbb{N}$, we obtain that

$$
\begin{aligned}
& \left\|\phi_{n}-\phi_{n+1}\right\|_{E_{0}}=\left\|\phi_{n}(c)-\phi_{n+1}(c)\right\|_{E} \\
= & \left\|T \phi_{n}-T \phi_{n-1}\right\|_{E}
\end{aligned}
$$




$$
\begin{aligned}
& \leq \psi\left(\max \left\{\left\|\phi_{n}-\phi_{n-1}\right\|_{E_{0}}, \frac{\left\|\phi_{n}(c)-T \phi_{n}\right\|_{E}\left\|\phi_{n-1}(c)-T \phi_{n-1}\right\|_{E}}{1+\left\|T \phi_{n}-T \phi_{n-1}\right\|_{E}}\right\}\right) \\
& =\psi\left(\max \left\{\left\|\phi_{n}-\phi_{n-1}\right\|_{E_{0}}, \frac{\left\|\phi_{n}(c)-\phi_{n+1}(c)\right\|_{E}\left\|\phi_{n-1}(c)-\phi_{n}(c)\right\|_{E}}{1+\left\|\phi_{n+1}(c)-\phi_{n}(c)\right\|_{E}}\right\}\right) \\
& \leq \psi\left(\max \left\{\left\|\phi_{n}-\phi_{n-1}\right\|_{E_{0}},\left\|\phi_{n-1}(c)-\phi_{n}(c)\right\|_{E}\right\}\right) \\
& =\psi\left(\max \left\{\left\|\phi_{n}-\phi_{n-1}\right\|_{E_{0}},\left\|\phi_{n-1}-\phi_{n}\right\|_{E_{0}}\right\}\right) \\
& =\psi\left(\left\|\phi_{n}-\phi_{n-1}\right\|_{E_{0}}\right) .
\end{aligned}
$$

By induction, we obtain that

$$
\left\|\phi_{n}-\phi_{n+1}\right\|_{E_{0}} \leq \psi^{n}\left(\left\|\phi_{0}-\phi_{1}\right\|_{E_{0}}\right) \text { for all } n \in \mathbb{N} .
$$

Fix $\varepsilon>0$. This implies that there exists $N \in \mathbb{N}$ such that

$$
\sum_{n \geq N} \psi^{n}\left(\left\|\phi_{0}-\phi_{1}\right\|_{E_{0}}\right)<\varepsilon .
$$

For each $m, n \in \mathbb{N}$ with $m>n>N$, we obtain that

$$
\begin{aligned}
\left\|\phi_{n}-\phi_{m}\right\|_{E_{0}} \leq \sum_{k=n}^{m-1}\left\|\phi_{k}-\phi_{k+1}\right\|_{E_{0}} \leq \sum_{k=n}^{m-1} \psi^{k}\left(\left\|\phi_{0}-\phi_{1}\right\|_{E_{0}}\right) & \\
& \leq \sum_{n \geq N} \psi^{n}\left(\left\|\phi_{0}-\phi_{1}\right\|_{E_{0}}<\varepsilon .\right.
\end{aligned}
$$

This implies that $\left\{\phi_{n}\right\}$ is a Cauchy sequence. By the completeness of $E_{0}$, we have $\lim _{n \rightarrow \infty} \phi_{n}=\phi$ for some $\phi \in E_{0}$ and

$$
\lim _{n \rightarrow \infty} T \phi_{n}=\lim _{n \rightarrow \infty} \phi_{n+1}(c)=\phi(c) .
$$

Since $\mathcal{R}_{c}$ is algebraically closed with respect to the norm topology, we have $\phi \in \mathcal{R}_{c}$. We next prove that $\phi$ is a PPF dependent fixed point of $T$. Using (4), we obtain that

$$
\begin{aligned}
& \|T \phi-\phi(c)\|_{E} \leq\left\|T \phi-\phi_{n}(c)\right\|_{E}+\left\|\phi_{n}(c)-\phi(c)\right\|_{E} \\
= & \left\|T \phi-T \phi_{n-1}\right\|_{E}+\left\|\phi_{n}(c)-\phi(c)\right\|_{E} \\
\leq & \psi\left(\max \left\{\left\|\phi-\phi_{n-1}\right\|_{E_{0}}, \frac{\|\phi(c)-T \phi\|_{E}\left\|\phi_{n-1}(c)-T \phi_{n-1}\right\|_{E}}{1+\left\|T \phi-T \phi_{n-1}\right\|_{E}}\right\}\right)+ \\
& \left\|\phi_{n}(c)-\phi(c)\right\|_{E}
\end{aligned}
$$




$$
\begin{aligned}
\leq & \psi\left(\max \left\{\left\|\phi-\phi_{n-1}\right\|_{E_{0}}, \frac{\|\phi(c)-T \phi\|_{E}\left\|\phi_{n-1}(c)-\phi_{n}(c)\right\|_{E}}{1+\left\|\phi_{n}(c)-T \phi\right\|_{E}}\right\}\right)+ \\
& \left\|\phi_{n}(c)-\phi(c)\right\|_{E},
\end{aligned}
$$

for all $n \in \mathbb{N}$. Since $\psi$ is continuous at 0 and

$$
\lim _{n \rightarrow \infty} \max \left\{\left\|\phi-\phi_{n-1}\right\|_{E_{0}}, \frac{\|\phi(c)-T \phi\|_{E}\left\|\phi_{n-1}(c)-\phi_{n}(c)\right\|_{E}}{1+\left\|\phi_{n}(c)-T \phi\right\|_{E}}\right\}=0,
$$

we have

$$
\lim _{n \rightarrow \infty} \psi\left(\max \left\{\left\|\phi-\phi_{n-1}\right\|_{E_{0}}, \frac{\|\phi(c)-T \phi\|_{E}\left\|\phi_{n-1}(c)-\phi_{n}(c)\right\|_{E}}{1+\left\|\phi_{n}(c)-T \phi\right\|_{E}}\right\}\right)=\psi(0)=0 .
$$

It follows that $\|T \phi-\phi(c)\|_{E}=0$ and therefore $T \phi=\phi(c)$. Hence $\phi$ is a PPF dependent fixed point of $T$ in $\mathcal{R}_{c}$. Suppose that $\alpha$ is any PPF dependent fixed point of $T$ in $\mathcal{R}_{c}$. Therefore

$$
\begin{aligned}
\|\phi-\alpha\|_{E_{0}} & =\|\phi(c)-\alpha(c)\|_{E} \\
& =\|T \phi-T \alpha\|_{E} \\
& \leq \psi\left(\max \left\{\|\phi-\alpha\|_{E_{0}}, \frac{\|\phi(c)-T \phi\|_{E}\|\alpha(c)-T \alpha\|_{E}}{1+\|T \phi-T \alpha\|_{E}}\right\}\right) \\
& =\psi\left(\|\phi-\alpha\|_{E_{0}}\right) .
\end{aligned}
$$

This implies that $\|\phi-\alpha\|_{E_{0}}=0$ and so $\phi=\alpha$. Hence $T$ has a unique PPF dependent fixed point of $T$ in $\mathcal{R}_{c}$.

We next prove the fixed point results for mappings satisfying generalized rational type contractions where $\psi \in \Psi_{2}$.

Theorem 9. Suppose that $\psi \in \Psi_{2}$ and $T: E_{0} \rightarrow E$ satisfies the following condition:

$$
\|T \phi-T \alpha\|_{E} \leq \psi\left(\max \left\{\|\phi-\alpha\|_{E_{0}}, \frac{\|\phi(c)-T \phi\|_{E}\|\alpha(c)-T \alpha\|_{E}}{1+\|T \phi-T \alpha\|_{E}}\right\}\right),
$$

for all $\phi, \alpha \in E_{0}$. Assume that $\mathcal{R}_{c}$ is topologically closed with respect to the norm topology and algebraically closed with respect to the difference. Then $T$ has a unique PPF dependent fixed point in $\mathcal{R}_{c}$.

Proof. As in the proof of Theorem 8, we can construct a sequence $\left\{\phi_{n}\right\}$ in $\mathcal{R}_{c}$ such that

$$
T \phi_{n}=\phi_{n+1}(c) \text { and }\left\|\phi_{n}-\phi_{n+1}\right\|_{E_{0}}=\left\|\phi_{n}(c)-\phi_{n+1}(c)\right\|_{E},
$$


for all $n \in \mathbb{N} \cup\{0\}$. Assume that $\phi_{n-1}=\phi_{n}$ for some $n \in \mathbb{N}$. It follows that $\phi_{n-1}(c)=\phi_{n}(c)=T \phi_{n-1}$. Therefore $T$ has a fixed point in $\mathcal{R}_{c}$. Suppose that $\phi_{n-1} \neq \phi_{n}$ for all $n \in \mathbb{N}$. For each $n \in \mathbb{N}$, we obtain that

$$
\begin{aligned}
& \left\|\phi_{n}-\phi_{n+1}\right\|_{E_{0}}=\left\|\phi_{n}(c)-\phi_{n+1}(c)\right\|_{E} \\
= & \left\|T \phi_{n}-T \phi_{n-1}\right\|_{E} \\
\leq & \psi\left(\max \left\{\left\|\phi_{n}-\phi_{n-1}\right\|_{E_{0}}, \frac{\left\|\phi_{n}(c)-T \phi_{n}\right\|_{E}\left\|\phi_{n-1}(c)-T \phi_{n-1}\right\|_{E}}{1+\left\|T \phi_{n}-T \phi_{n-1}\right\|_{E}}\right\}\right) \\
= & \psi\left(\max \left\{\left\|\phi_{n}-\phi_{n-1}\right\|_{E_{0}}, \frac{\left\|\phi_{n}(c)-\phi_{n+1}(c)\right\|_{E}\left\|\phi_{n-1}(c)-\phi_{n}(c)\right\|_{E}}{1+\left\|\phi_{n+1}(c)-\phi_{n}(c)\right\|_{E}}\right\}\right) \\
\leq & \psi\left(\max \left\{\left\|\phi_{n}-\phi_{n-1}\right\|_{E_{0}},\left\|\phi_{n-1}(c)-\phi_{n}(c)\right\|_{E}\right\}\right) \\
= & \psi\left(\max \left\{\left\|\phi_{n}-\phi_{n-1}\right\|_{E_{0}},\left\|\phi_{n-1}-\phi_{n}\right\|_{E_{0}}\right\}\right) \\
= & \psi\left(\left\|\phi_{n}-\phi_{n-1}\right\|_{E_{0}}\right) \\
< & \left\|\phi_{n}-\phi_{n-1}\right\|_{E_{0}} .
\end{aligned}
$$

It follows that $\left\{\left\|\phi_{n}-\phi_{n+1}\right\|_{E_{0}}\right\}$ is a nonincreasing sequence of nonnegative real numbers and so it is a convergent sequence. Suppose that

$$
\lim _{n \rightarrow \infty}\left\|\phi_{n}-\phi_{n+1}\right\|_{E_{0}}=\alpha,
$$

for some nonnegative real number $\alpha$. We will prove that $\alpha=0$. Suppose that $\alpha>0$. Since

$$
\left\|\phi_{n}-\phi_{n+1}\right\|_{E_{0}} \leq \psi\left(\left\|\phi_{n}-\phi_{n-1}\right\|_{E_{0}}\right),
$$

for all $n \in \mathbb{N}$ and by the continuity of $\psi$, we have $\alpha \leq \psi(\alpha)<\alpha$ which leads to a contradiction. This implies that $\alpha=0$. We next prove that the sequence $\left\{\phi_{n}\right\}$ is a Cauchy sequence in $E_{0}$. Assume that $\left\{\phi_{n}\right\}$ is not a Cauchy sequence. It follows that there exist $\varepsilon>0$ and two sequences of positive integers $\left\{m_{k}\right\}$ and $\left\{n_{k}\right\}$ satisfying $m_{k}>n_{k}>k$ for each $k \in \mathbb{N}$ and

$$
\left\|\phi_{m_{k}}-\phi_{n_{k}}\right\|_{E_{0}} \geq \varepsilon .
$$

Let $\left\{m_{k}\right\}$ be the sequence of the least positive integers exceeding $\left\{n_{k}\right\}$ which satisfies (6) and

$$
\left\|\phi_{m_{k}-1}-\phi_{n_{k}}\right\|_{E_{0}}<\varepsilon .
$$

We will prove that $\lim _{k \rightarrow \infty}\left\|\phi_{m_{k}}-\phi_{n_{k}}\right\|_{E_{0}}=\varepsilon$. Since $\left\|\phi_{m_{k}}-\phi_{n_{k}}\right\|_{E_{0}} \geq \varepsilon$ for all $k \in \mathbb{N}$, we have

$$
\lim _{k \rightarrow \infty}\left\|\phi_{m_{k}}-\phi_{n_{k}}\right\|_{E_{0}} \geq \varepsilon .
$$


For each $k \in \mathbb{N}$, we obtain that

$$
\begin{aligned}
\left\|\phi_{m_{k}}-\phi_{n_{k}}\right\|_{E_{0}} & \leq\left\|\phi_{m_{k}}-\phi_{m_{k}-1}\right\|_{E_{0}}+\left\|\phi_{m_{k}-1}-\phi_{n_{k}}\right\|_{E_{0}} \\
& \leq\left\|\phi_{m_{k}}-\phi_{m_{k}-1}\right\|_{E_{0}}+\varepsilon .
\end{aligned}
$$

This implies that $\lim _{k \rightarrow \infty}\left\|\phi_{m_{k}}-\phi_{n_{k}}\right\|_{E_{0}} \leq \varepsilon$. Therefore

$$
\lim _{k \rightarrow \infty}\left\|\phi_{m_{k}}-\phi_{n_{k}}\right\|_{E_{0}}=\varepsilon .
$$

Similarly, we can prove that

$$
\lim _{k \rightarrow \infty}\left\|\phi_{m_{k}+1}-\phi_{n_{k}}\right\|_{E_{0}}=\varepsilon, \lim _{k \rightarrow \infty}\left\|\phi_{m_{k}}-\phi_{n_{k}-1}\right\|_{E_{0}}=\varepsilon,
$$

and

$$
\lim _{k \rightarrow \infty}\left\|\phi_{m_{k}+1}-\phi_{n_{k}-1}\right\|_{E_{0}}=\varepsilon .
$$

Since $\mathcal{R}_{C}$ is algebraically closed with respect to the difference, for each $k \in \mathbb{N}$, we obtain that

$$
\begin{aligned}
\left\|\phi_{n_{k}}-\phi_{m_{k}+1}\right\|_{E_{0}}= & \left\|\phi_{n_{k}}(c)-\phi_{m_{k}+1}(c)\right\|_{E} \\
= & \left\|T \phi_{m_{k}}-T \phi_{n_{k}-1}\right\|_{E} \\
\leq & \psi\left(\operatorname { m a x } \left\{\left\|\phi_{m_{k}}-\phi_{n_{k}-1}\right\|_{E_{0}},\right.\right. \\
& \left.\left.\frac{\left\|\phi_{m_{k}}(c)-T \phi_{m_{k}}\right\|_{E}\left\|\phi_{n_{k}-1}(c)-T \phi_{n_{k}-1}\right\|_{E}}{1+\left\|T \phi_{n_{k}-1}-T \phi_{m_{k}}\right\|_{E}}\right\}\right) \\
= & \psi\left(\operatorname { m a x } \left\{\left\|\phi_{m_{k}}-\phi_{n_{k}-1}\right\|_{E_{0}},\right.\right. \\
& \left.\left.\frac{\left\|\phi_{m_{k}}(c)-\phi_{m_{k}+1}(c)\right\|_{E}\left\|\phi_{n_{k}-1}(c)-\phi_{n_{k}}(c)\right\|_{E}}{1+\left\|\phi_{n_{k}}(c)-\phi_{m_{k}+1}(c)\right\|_{E}}\right\}\right) .
\end{aligned}
$$

By taking the limit of both sides, we have

$$
\varepsilon \leq \psi(\varepsilon)<\varepsilon
$$

which leads to a contradiction. It follows that the sequence $\left\{\phi_{n}\right\}$ is a Cauchy sequence. By the completeness of $E_{0}$, we have $\lim _{n \rightarrow \infty} \phi_{n}=\phi$ for some $\phi \in E_{0}$ and

$$
\lim _{n \rightarrow \infty} T \phi_{n}=\lim _{n \rightarrow \infty} \phi_{n+1}(c)=\phi(c) .
$$

Since $\mathcal{R}_{c}$ is algebraically closed with respect to the norm topology, we have $\phi \in \mathcal{R}_{c}$. We will prove that $\phi$ is a PPF dependent fixed point of $T$. Using (5), we obtain that

$$
\|T \phi-\phi(c)\|_{E} \leq\left\|T \phi-\phi_{n}(c)\right\|_{E}+\left\|\phi_{n}(c)-\phi(c)\right\|_{E}
$$




$$
\begin{aligned}
= & \left\|T \phi-T \phi_{n-1}\right\|_{E}+\left\|\phi_{n}(c)-\phi(c)\right\|_{E} \\
\leq & \psi\left(\max \left\{\left\|\phi-\phi_{n-1}\right\|_{E_{0}}, \frac{\|\phi(c)-T \phi\|_{E}\left\|\phi_{n-1}(c)-T \phi_{n-1}\right\|_{E}}{1+\left\|T \phi_{n-1}-T \phi\right\|_{E}}\right\}\right)+ \\
& \left\|\phi_{n}(c)-\phi(c)\right\|_{E} \\
= & \psi\left(\max \left\{\left\|\phi-\phi_{n-1}\right\|_{E_{0}} \frac{\|\phi(c)-T \phi\|_{E}\left\|\phi_{n-1}(c)-\phi_{n}(c)\right\|_{E}}{1+\left\|\phi_{n}(c)-T \phi\right\|_{E}}\right\}\right)+ \\
& \left\|\phi_{n}(c)-\phi(c)\right\|_{E},
\end{aligned}
$$

for all $n \in \mathbb{N}$. Taking the limit as $n \rightarrow \infty$, since $\psi$ is continuous, we obtain that $\|T \phi-\phi(c)\|_{E}=0$ and therefore $T \phi=\phi(c)$. Hence $\phi$ is a PPF dependent fixed point of $T$. Suppose that $\alpha$ is any PPF dependent fixed point of $T$ in $\mathcal{R}_{c}$. Therefore

$$
\begin{aligned}
\|\phi-\alpha\|_{E_{0}} & \leq\|\phi(c)-\alpha(c)\|_{E} \\
& =\|T \phi-T \alpha\|_{E} \\
& \leq \psi\left(\max \left\{\|\phi-\alpha\|_{E_{0}}, \frac{\|\phi(c)-T \phi\|_{E}\|\alpha(c)-T \alpha\|_{E}}{1+\|T \phi-T \alpha\|_{E}}\right\}\right) \\
& =\psi\left(\|\phi-\alpha\|_{E_{0}}\right) .
\end{aligned}
$$

This implies that $\|\phi-\alpha\|_{E_{0}}=0$ and so $\phi=\alpha$. Hence $T$ has a unique PPF dependent fixed point of $T$ in $\mathcal{R}_{c}$.

By applying Theorem 9, we obtain the following corollary.

Corollary 10. Suppose that $\psi \in \Psi_{2}$ and $T: E_{0} \rightarrow E$ satisfies the following condition:

$$
\|T \phi-T \alpha\|_{E} \leq k \max \left\{\|\phi-\alpha\|_{E_{0}}, \frac{\|\phi(c)-T \phi\|_{E}\|\alpha(c)-T \alpha\|_{E}}{1+\|T \phi-T \alpha\|_{E}}\right\},
$$

for all $\phi, \alpha \in E_{0}$ where $k \in[0,1)$. Assume that $\mathcal{R}_{c}$ is topologically closed with respect to the norm topology and algebraically closed with respect to the difference. Then $T$ has a unique PPF dependent fixed point in $\mathcal{R}_{c}$.

Proof. Define a function $\psi:[0,+\infty) \rightarrow[0,+\infty)$ by $\psi(t)=k t$ for all $t \in$ $[0,+\infty)$. Thus $\psi$ is a continuous nondecreasing mapping and

$$
\psi(t)<t \text {, for all } t \in(0,+\infty) .
$$

It follows that all assumptions in Theorem 9 are satisfied. Hence the proof is complete. 
We immediately obtain the following corollary proved by Sintunavarat and Kumam [18].

Corollary 11. [18] Suppose that $T: E_{0} \rightarrow E$ is a rational type contraction. If $\mathcal{R}_{c}$ is topologically closed with respect to the norm topology and algebraically closed with respect to the difference, then $T$ has a unique PPF dependent fixed point in $\mathcal{R}_{c}$.

\section{PPF Dependent Fixed Points for Rational Type Contractions in Partially Ordered Banach Spaces}

In this section, we prove the fixed point results for mappings satisfying the generalized contractive conditions in a partially ordered Banach space $(E, \leq)$.

Theorem 12. Suppose that $\psi \in \Psi_{1}$ and $T: E_{0} \rightarrow E$ satisfies the following conditions:

(i) $T$ is a nondecreasing mapping;

(ii) for all $\phi, \alpha \in E_{0}$ with $\alpha \leq \phi$, we have

$$
\|T \phi-T \alpha\|_{E} \leq \psi\left(\max \left\{\|\phi-\alpha\|_{E_{0}}, \frac{\|\phi(c)-T \phi\|_{E}\|\alpha(c)-T \alpha\|_{E}}{1+\|T \phi-T \alpha\|_{E}}\right\}\right)
$$

(iii) there exists a lower solution $\phi_{0} \in \mathcal{R}_{c}$ such that $\phi_{0}(c) \leq T \phi_{0}$;

(iv) $T$ is a continuous mapping.

Assume that $\mathcal{R}_{c}$ is topologically closed with respect to the norm topology and algebraically closed with respect to the difference. Then $T$ has a PPF dependent fixed point in $\mathcal{R}_{c}$.

Proof. Since $\phi_{0} \in \mathcal{R}_{c}$ and $T \phi_{0} \in E$, there exists $x_{1} \in E$ such that $T \phi_{0}=x_{1}$. Choose $\phi_{1} \in \mathcal{R}_{c}$ such that $x_{1}=\phi_{1}(c)$. Since $\phi_{0} \in \mathcal{R}_{c}$ is a lower solution such that $\phi_{0}(c) \leq T \phi_{0}$, we obtain that $\phi_{0} \leq \phi_{1}$. By the algebraic closedness with respect to difference of $\mathcal{R}_{c}$, we have

$$
\left\|\phi_{0}-\phi_{1}\right\|_{E_{0}}=\left\|\phi_{0}(c)-\phi_{1}(c)\right\|_{E} .
$$

Using the fact that $T$ is nondecreasing, we obtain that

$$
\phi_{1}(c)=T \phi_{0} \leq T \phi_{1}
$$


By continuing the process as before, we can construct the sequence $\left\{\phi_{n}\right\}$ in $\mathcal{R}_{c}$ such that

$$
\begin{gathered}
T \phi_{0}=\phi_{1}(c) \leq T \phi_{1}=\phi_{2}(c) \leq \cdots \leq T \phi_{n}=\phi_{n+1}(c) \leq T \phi_{n+1} \cdots, \\
\phi_{n} \leq \phi_{n+1} \text { and }\left\|\phi_{n}-\phi_{n+1}\right\|_{E_{0}}=\left\|\phi_{n}(c)-\phi_{n+1}(c)\right\|_{E}
\end{gathered}
$$

for all $n \in \mathbb{N} \cup\{0\}$. For each $n \in \mathbb{N}$, we obtain that

$$
\begin{aligned}
& \left\|\phi_{n}-\phi_{n+1}\right\|_{E_{0}}=\left\|\phi_{n}(c)-\phi_{n+1}(c)\right\|_{E} \\
= & \left\|T \phi_{n}-T \phi_{n-1}\right\|_{E} \\
\leq & \psi\left(\max \left\{\left\|\phi_{n}-\phi_{n-1}\right\|_{E_{0}}, \frac{\left\|\phi_{n}(c)-T \phi_{n}\right\|_{E}\left\|\phi_{n-1}(c)-T \phi_{n-1}\right\|_{E}}{1+\left\|T \phi_{n}-T \phi_{n-1}\right\|_{E}}\right)\right. \\
= & \psi\left(\max \left\{\left\|\phi_{n}-\phi_{n-1}\right\|_{E_{0}}, \frac{\left\|\phi_{n}(c)-\phi_{n+1}(c)\right\|_{E}\left\|\phi_{n-1}(c)-\phi_{n}(c)\right\|_{E}}{1+\left\|\phi_{n+1}(c)-\phi_{n}(c)\right\|_{E}}\right\}\right) \\
\leq & \psi\left(\max \left\{\left\|\phi_{n}-\phi_{n-1}\right\|_{E_{0}},\left\|\phi_{n-1}(c)-\phi_{n}(c)\right\|_{E}\right\}\right) \\
= & \psi\left(\max \left\{\left\|\phi_{n}-\phi_{n-1}\right\|_{E_{0}},\left\|\phi_{n-1}-\phi_{n}\right\|_{E_{0}}\right\}\right) \\
= & \psi\left(\left\|\phi_{n}-\phi_{n-1}\right\|_{E_{0}}\right) .
\end{aligned}
$$

By induction, we obtain that

$$
\left\|\phi_{n}-\phi_{n+1}\right\|_{E_{0}} \leq \psi^{n}\left(\left\|\phi_{0}-\phi_{1}\right\|_{E_{0}} .\right.
$$

By the analogous proof as in Theorem 8, we have $\left\{\phi_{n}\right\}$ is a Cauchy sequence. By the completeness of $E_{0}$, we have $\lim _{n \rightarrow \infty} \phi_{n}=\phi$ for some $\phi \in E_{0}$ and

$$
\lim _{n \rightarrow \infty} T \phi_{n}=\lim _{n \rightarrow \infty} \phi_{n+1}(c)=\phi(c) .
$$

Since $\mathcal{R}_{c}$ is algebraically closed with respect to the norm topology, we have $\phi \in \mathcal{R}_{c}$. Using the continuity of $T$, we obtain that $\lim _{n \rightarrow \infty} T \phi_{n}=T \phi$. By the uniqueness of the limit, we have $T \phi=\phi(c)$. Hence $\phi$ is a PPF dependent fixed point of $T$.

Theorem 13. Suppose that $\psi \in \Psi_{1}$ and $T: E_{0} \rightarrow E$ satisfies the following conditions:

(i) $T$ is a nondecreasing mapping;

(ii) for all $\phi, \alpha \in E_{0}$ with $\alpha \leq \phi$, we have

$$
\|T \phi-T \alpha\|_{E} \leq \psi\left(\max \left\{\|\phi-\alpha\|_{E_{0}}, \frac{\|\phi(c)-T \phi\|_{E}\|\alpha(c)-T \alpha\|_{E}}{1+\|T \phi-T \alpha\|_{E}}\right\}\right) ;
$$


(iii) there exists a lower solution $\phi_{0} \in \mathcal{R}_{c}$ such that $\phi_{0}(c) \leq T \phi_{0}$;

(iv) if $\left\{\phi_{n}\right\}$ is a nondecreasing sequence in $E_{0}$ converging to $\phi \in E_{0}$, then $\phi_{n} \leq \phi$ for all $n \in \mathbb{N}$.

Assume that $\mathcal{R}_{c}$ is topologically closed with respect to the norm topology and algebraically closed with respect to the difference. Then $T$ has a PPF dependent fixed point in $\mathcal{R}_{c}$.

Proof. As in Theorem 12, we construct a nondecreasing sequence $\left\{\phi_{n}\right\}$ in $\mathcal{R}_{c}$ converging to $\phi \in \mathcal{R}_{c}$. This implies that $\phi_{n} \leq \phi$ for all $n \in \mathbb{N}$. Therefore

$$
\begin{aligned}
& \|T \phi-\phi(c)\|_{E} \leq\left\|T \phi-\phi_{n}(c)\right\|_{E}+\left\|\phi_{n}(c)-\phi(c)\right\|_{E} \\
= & \left\|T \phi-T \phi_{n-1}\right\|_{E}+\left\|\phi_{n}(c)-\phi(c)\right\|_{E} \\
\leq & \psi\left(\max \left\{\left\|\phi-\phi_{n-1}\right\|_{E_{0}}, \frac{\|\phi(c)-T \phi\|_{E}\left\|\phi_{n-1}(c)-T \phi_{n-1}\right\|_{E}}{1+\left\|T \phi_{n-1}-T \phi\right\|_{E}}\right\}\right)+ \\
& \left\|\phi_{n}(c)-\phi(c)\right\|_{E} \\
\leq & \psi\left(\max \left\{\left\|\phi-\phi_{n-1}\right\|_{E_{0}}, \frac{\|\phi(c)-T \phi\|_{E}\left\|\phi_{n-1}(c)-\phi_{n}(c)\right\|_{E}}{1+\left\|\phi_{n}(c)-T \phi\right\|_{E}}\right\}\right)+ \\
& \left\|\phi_{n}(c)-\phi(c)\right\|_{E},
\end{aligned}
$$

for all $n \in \mathbb{N}$. Since $\psi$ is continuous at 0 and

$$
\lim _{n \rightarrow \infty} \max \left\{\left\|\phi-\phi_{n-1}\right\|_{E_{0}}, \frac{\|\phi(c)-T \phi\|_{E}\left\|\phi_{n-1}(c)-\phi_{n}(c)\right\|_{E}}{1+\left\|\phi_{n}(c)-T \phi\right\|_{E}}\right\}=0,
$$

we have

$$
\lim _{n \rightarrow \infty} \psi\left(\max \left\{\left\|\phi-\phi_{n-1}\right\|_{E_{0}}, \frac{\|\phi(c)-T \phi\|_{E}\left\|\phi_{n-1}(c)-\phi_{n}(c)\right\|_{E}}{1+\left\|\phi_{n}(c)-T \phi\right\|_{E}}\right\}\right)=\psi(0)=0 .
$$

It follows that $\|T \phi-\phi(c)\|_{E}=0$ and therefore $T \phi=\phi(c)$. Hence $\phi$ is a PPF dependent fixed point of $T$.

Theorem 14. Suppose that $\psi \in \Psi_{2}$ and $T: E_{0} \rightarrow E$ satisfies the following conditions:

(i) $T$ is a nondecreasing mapping;

(ii) for all $\phi, \alpha \in E_{0}$ with $\alpha \leq \phi$, we have

$$
\|T \phi-T \alpha\|_{E} \leq \psi\left(\max \left\{\|\phi-\alpha\|_{E_{0}}, \frac{\|\phi(c)-T \phi\|_{E}\|\alpha(c)-T \alpha\|_{E}}{1+\|T \phi-T \alpha\|_{E}}\right\}\right)
$$


(iii) there exists a lower solution $\phi_{0} \in \mathcal{R}_{c}$ such that $\phi_{0}(c) \leq T \phi_{0}$;

(iv) $T$ is a continuous mapping.

Assume that $\mathcal{R}_{c}$ is topologically closed with respect to the norm topology and algebraically closed with respect to the difference. Then $T$ has a PPF dependent fixed point in $\mathcal{R}_{c}$.

Proof. As in Theorem 12, can we construct a sequence $\left\{\phi_{n}\right\}$ in $\mathcal{R}_{c}$ such that

$$
\begin{gathered}
T \phi_{0}=\phi_{1}(c) \leq T \phi_{1}=\phi_{2}(c) \leq \cdots \leq T \phi_{n}=\phi_{n+1}(c) \leq T \phi_{n+1} \cdots \\
\phi_{n} \leq \phi_{n+1} \text { and }\left\|\phi_{n}-\phi_{n+1}\right\|_{E_{0}}=\left\|\phi_{n}(c)-\phi_{n+1}(c)\right\|_{E}
\end{gathered}
$$

for all $n \in \mathbb{N} \cup\{0\}$. Assume that $\phi_{n-1}=\phi_{n}$ for some $n \in \mathbb{N}$. It follows that $\phi_{n-1}(c)=\phi_{n}(c)=T \phi_{n-1}$. Therefore $T$ has a fixed point in $\mathcal{R}_{c}$. Suppose that $\phi_{n-1} \neq \phi_{n}$ for all $n \in \mathbb{N}$. For each $n \in \mathbb{N}$, we obtain that

$$
\begin{aligned}
& \left\|\phi_{n}-\phi_{n+1}\right\|_{E_{0}}=\left\|\phi_{n}(c)-\phi_{n+1}(c)\right\|_{E} \\
= & \left\|T \phi_{n}-T \phi_{n-1}\right\|_{E} \\
\leq & \psi\left(\max \left\{\left\|\phi_{n}-\phi_{n-1}\right\|_{E_{0}}, \frac{\left\|\phi_{n}(c)-T \phi_{n}\right\|_{E}\left\|\phi_{n-1}(c)-T \phi_{n-1}\right\|_{E}}{1+\left\|T \phi_{n}-T \phi_{n-1}\right\|_{E}}\right)\right. \\
= & \psi\left(\max \left\{\left\|\phi_{n}-\phi_{n-1}\right\|_{E_{0}}, \frac{\left\|\phi_{n}(c)-\phi_{n+1}(c)\right\|_{E}\left\|\phi_{n-1}(c)-\phi_{n}(c)\right\|_{E}}{1+\left\|\phi_{n+1}(c)-\phi_{n}(c)\right\|_{E}}\right\}\right) \\
\leq & \psi\left(\max \left\{\left\|\phi_{n}-\phi_{n-1}\right\|_{E_{0}},\left\|\phi_{n-1}(c)-\phi_{n}(c)\right\|_{E}\right\}\right) \\
= & \psi\left(\max \left\{\left\|\phi_{n}-\phi_{n-1}\right\|_{E_{0}},\left\|\phi_{n-1}-\phi_{n}\right\|_{E_{0}}\right\}\right) \\
= & \psi\left(\left\|\phi_{n}-\phi_{n-1}\right\|_{E_{0}}\right) \\
< & \left\|\phi_{n}-\phi_{n-1}\right\|_{E_{0}} .
\end{aligned}
$$

By an analogous proof as in Theorem 9, we obtain that

$$
\lim _{n \rightarrow \infty}\left\|\phi_{n}-\phi_{n+1}\right\|_{E_{0}}=0 \text { and } \lim _{n \rightarrow \infty} \phi_{n}=\phi \text { for some } \phi \in \mathcal{R}_{c}
$$

Therefore

$$
\lim _{n \rightarrow \infty} T \phi_{n}=\lim _{n \rightarrow \infty} \phi_{n+1}(c)=\phi(c) .
$$

Using the continuity of $T$, we obtain that $\lim _{n \rightarrow \infty} T \phi_{n}=T \phi$. By the uniqueness of the limit, we have $T \phi=\phi(c)$. Hence $\phi$ is a PPF dependent fixed point of $T$. 
Theorem 15. Suppose that $\psi \in \Psi_{2}$ and $T: E_{0} \rightarrow E$ satisfies the following conditions:

(i) $T$ is a nondecreasing mapping;

(ii) for all $\phi, \alpha \in E_{0}$ with $\alpha \leq \phi$, we have

$$
\|T \phi-T \alpha\|_{E} \leq \psi\left(\max \left\{\|\phi-\alpha\|_{E_{0}}, \frac{\|\phi(c)-T \phi\|_{E}\|\alpha(c)-T \alpha\|_{E}}{1+\|T \phi-T \alpha\|_{E}}\right\}\right)
$$

(iii) there exists a lower solution $\phi_{0} \in \mathcal{R}_{c}$ such that $\phi_{0}(c) \leq T \phi_{0}$;

(iv) if $\left\{\phi_{n}\right\}$ is a nondecreasing sequence in $E_{0}$ converging to $\phi \in E_{0}$, then $\phi_{n} \leq \phi$ for all $n \in \mathbb{N}$.

Assume that $\mathcal{R}_{c}$ is topologically closed with respect to the norm topology and algebraically closed with respect to the difference. Then $T$ has a PPF dependent fixed point in $\mathcal{R}_{c}$.

Proof. As in Theorem 14, we can construct a nondecreasing sequence $\left\{\phi_{n}\right\}$ in $\mathcal{R}_{c}$ converging to $\phi \in \mathcal{R}_{c}$. This implies that $\phi_{n} \leq \phi$ for all $n \in \mathbb{N}$. Therefore

$$
\begin{aligned}
& \|T \phi-\phi(c)\|_{E} \leq\left\|T \phi-\phi_{n}(c)\right\|_{E}+\left\|\phi_{n}(c)-\phi(c)\right\|_{E} \\
= & \left\|T \phi-T \phi_{n-1}\right\|_{E}+\left\|\phi_{n}(c)-\phi(c)\right\|_{E} \\
\leq & \psi\left(\max \left\{\left\|\phi-\phi_{n-1}\right\|_{E_{0}}, \frac{\|\phi(c)-T \phi\|_{E}\left\|\phi_{n-1}(c)-T \phi_{n-1}\right\|_{E}}{1+\left\|T \phi_{n-1}-T \phi\right\|_{E}}\right\}\right)+ \\
& \left\|\phi_{n}(c)-\phi(c)\right\|_{E} \\
\leq & \psi\left(\max \left\{\left\|\phi-\phi_{n-1}\right\|_{E_{0}}, \frac{\|\phi(c)-T \phi\|_{E}\left\|\phi_{n-1}(c)-\phi_{n}(c)\right\|_{E}}{1+\left\|\phi_{n}(c)-T \phi\right\|_{E}}\right\}\right)+ \\
& \left\|\phi_{n}(c)-\phi(c)\right\|_{E},
\end{aligned}
$$

for all $n \in \mathbb{N}$. Taking the limit as $n \rightarrow \infty$, since $\psi$ is continuous, we obtain that $\|T \phi-\phi(c)\|_{E}=0$ and therefore $T \phi=\phi(c)$. Hence $\phi$ is a PPF dependent fixed point of $T$.

\section{Acknowledgments}

This research is supported by Naresuan University, Thailand under grant R255 $7 \mathrm{~B} 055$. 


\section{References}

[1] I. Beg and M. Abbas, Coincidence point and invariant approximation for mappings satisfying generalized weak contractive condition, Fixed Point Theory Appl., (2006), doi:10.1155/2006/74503.

[2] S.R. Bernfeld, V. Lakshmikatham, Y.M. Reddy, Fixed point theorems of operators with PPF dependence in Banach spaces, Applicable Anal., 6 (1977), 271-280.

[3] B.K. Dass and S. Gupta, An extension of Banach contraction principle through rational expression, Indian J. Pure Appl. Math., 6 (1975), 14551458.

[4] B.C. Dhage, On some common fixed point theorems with PPF dependence in Banach spaces, J. Nonlinear Sci. Appl., 5 (2012), 220-232.

[5] Z. Drici, F.A. McRae and J. Vasundhara Devi, Fixed point theorems in partially ordered metric spaces for operators with PPF dependence, Nonlinear Anal. 67 (2007), 641-647.

[6] Z. Drici, F.A. McRae and J. Vasundhara Devi, Fixed point theorems for mixed monotone operators with PPF dependence, Nonlinear Anal., 69 (2008), 632-636.

[7] D.S. Jaggi, Some unique fixed point theorems, Indian J. Pure Appl. Math., 8 (1977), 223-230.

[8] G. Jungck,Compatible mappings and common fixed points, Int. J. Math. Sci., 9 (1968), 771-779.

[9] G. Jungck, Common fixed points for commuting and compatible maps on compacta, Proc. Amer. Math. Soc., 103 (1988), 977-983.

[10] G. Jungck, Common fixed points for noncontinuous nonself maps on nonmetric spaces, Far East J. Math. Sci., 4 (1996), 199-215.

[11] G. Jungck and N. Hussain, Compatible maps and invariant approximations, J. Math. Model. Algorithms., 325 (2007), 1003-1012.

[12] A. Kaewcharoen, PPF dependent common fixed point theorems for mappings in Banach spaces, J. Inequal. Appl., 2013 (2013), doi:10.1186/1029242X-2013-287. 
[13] R.P Pant, Common fixed points of noncommuting mappings, J. Math. Anal. Appl., 188 (1994), 436-440.

[14] V. Pant, Common fixed points under Lipschitz type condition, Bull. Korean Math. Soc., 45 (2008) No. 3, 467-475.

[15] H.L. Royden, Real Analysis, 3rd ed., Prentice Hall International Inc. (1988).

[16] B. Samet, C. Vetro and P. Vetro, Fixed point theorems for $\alpha$ - $\psi$-contractive type mappings, Nonlinear Anal., 75 (2012), 2154-2165.

[17] W. Sintunavarat and P. Kumam, Common fixed point theorems for generalized $\mathcal{J} \mathcal{H}$-operators classed and invariant approximations, J. Inequal. Appl., (2011). doi:10.1186/1029-242X-2011-67.

[18] W. Sintunavarat and P. Kumam, PPF dependent fixed point theorems for rational type contractive mappings in Banach spaces, J. Nonlinear Anal. Opt., 4 (2013), 157-162. 